\title{
Hubble Space Telescope Spectroscopic Observations of the Ejecta of SN 1987A at 2000 Days
}

\section{Citation}

Wang, Lifan, J. Craig Wheeler, Robert P. Kirshner, Peter M. Challis, Alexei V. Filippenko, Claes Fransson, Nino Panagia, Mark M. Phillips, and Nicholas Suntzeff. 1996. "Hubble Space Telescope Spectroscopic Observations of the Ejecta of SN 1987A at 2000 Days." The Astrophysical Journal 466 (August): 998. https://doi.org/10.1086/177570.

\section{Permanent link}

http://nrs.harvard.edu/urn-3:HUL.InstRepos:41399874

\section{Terms of Use}

This article was downloaded from Harvard University's DASH repository, and is made available under the terms and conditions applicable to Other Posted Material, as set forth at http:// nrs.harvard.edu/urn-3:HUL.InstRepos:dash.current.terms-of-use\#LAA

\section{Share Your Story}

The Harvard community has made this article openly available.

Please share how this access benefits you. Submit a story.

\section{Accessibility}




\title{
Hubble Space Telescope Spectroscopic Observations of the Ejecta of SN 1987A at 2000 Days
}

\author{
Lifan Wang], J. Craig Wheeler \\ Dept. of Astronomy and McDonald Observatory, The University of Texas at Austin, \\ Austin, TX 78712 \\ Robert P. Kirshner, Peter M. Challis \\ Harvard-Smithsonian Center for Astrophysics, 60 Garden Street, MS-19, Cambridge, MA \\ 02138 \\ Alexei V. Filippenko \\ Department of Astronomy, University of California, Berkeley, CA 94720 \\ Claes Fransson \\ Stockholm Observatory, S-133 00 Saltsjöbaden, Sweden \\ Nino Panagia \\ Space Telescope Science Institute, 3700 San Martin Drive, Baltimore, MD 21218 \\ and \\ Mark M. Phillips, Nicholas Suntzeff \\ Cerro Tololo Inter-American Observatory, National Optical Astronomy Observatories, \\ Casilla 603, La Serena, Chile
}

\begin{abstract}
We have used the Faint Object Spectrograph on the Hubble Space Telescope to observe the spectra of SN 1987A over the wavelength range $2000-8000$ $\AA$ on dates 1862 and 2210 days after the supernova outburst. Even these pre-COSTAR observations avoid much of the contamination from the bright stars nearby and provide a very useful set of line strengths and shapes for

\footnotetext{
${ }^{1}$ Based on observations with the NASA/ESA Hubble Space Telescope, obtained at the Space Telescope Science Institute, which is operated by AURA, inc., under NASA contract NAS 5-26555

${ }^{2}$ Also Beijing Astronomical Observatory, Beijing 100080, P. R. China

${ }^{3}$ Affiliated to the Astrophysics Division, Space Science Department of ESA.
} 
analysis. The spectrum is formed in an unusual physical setting: cold gas which is excited and ionized by energetic electrons from the radioactive debris of the supernova explosion. The spectra of SN 1987A at this phase are surprisingly similar to those of the nova shells of CP Puppis and T Pyxidis decades after outburst. SN 1987A and the novae are characterized by emission from material with electron temperatures of only a few hundred degrees Kelvin, and show narrow Balmer continuum emission and strong emission lines from $\mathrm{O}^{+}$. The Balmer continuum shape requires the electron temperature in the supernova ejecta to be as low as $500 \mathrm{~K}$ on day 1862 and $400 \mathrm{~K}$ on day 2210 after outburst. The [O II] $\lambda \lambda 3726,3728$ doublet is surprisingly strong and is plausibly powered by collisional ionization of neutral oxygen to excited states of $\mathrm{O}^{+}$.

The line intensity ratio of the [O I] $\lambda \lambda 6300,6364$ doublet obtained from Gaussian fits of the line profiles is $1.8 \pm 0.2$, contrary to the optically thin limit of 3. This ratio is not due to an optical depth effect, but rather is an artifact of assuming a Gaussian profile to fit the $\left[\begin{array}{ll}\mathrm{O} & \mathrm{I}\end{array}\right] \lambda \lambda 6300,6364$ doublet profile. Specifying the line ratio $R=F([\mathrm{OI}] 6300) / F([\mathrm{OI}] 6364)=3$ is consistent with the data and allows a calculation of the decomposed line profile.

All the observed strong lines are found to be blueshifted by a similar amount

of $400 \mathrm{~km} \mathrm{~s}^{-1}$. The line profiles are quite similar for lines arising from different chemical elements. The profiles are all asymmetric, showing redshifted extended tails with velocities up to $10,000 \mathrm{~km} \mathrm{~s}^{-1}$ in some strong lines. The blueshift of the line peaks is attributed to dust that condensed from the SN 1987A ejecta which is still distributed in dense opaque clumps. The strongest ultraviolet lines are those of $\mathrm{Mg}$ I $\lambda 2852$ and Mg II $\lambda \lambda 2795,2802$. The Mg I $\lambda 2852$ line is significantly broader than most lines in the optical, which provides a natural explanation for the size differences in the optical and ultraviolet of the SN 1987A ejecta derived from HST direct images.

Subject headings: stars: individual (SN 1987A) - stars: supernovae

\section{Introduction}

SN 1987A provides a unique opportunity to observe the debris from a supernova explosion. As part of our Supernova INtensive Study (SINS) of supernovae with HST, we present spectroscopic observations obtained with the Hubble Space Telescope (HST). When these HST observations were taken, in 1992 and 1993, the ejecta were so cold that 
thermal electrons played a negligible role in the formation of optical and ultraviolet lines. The observed emission lines are powered through non-thermal excitations by fast electrons produced by collisions with the gamma rays that result from the decay of radioactive

${ }^{57} \mathrm{Co}$ and ${ }^{44} \mathrm{Ti}$ produced in the supernova explosion. Detailed models for the spectroscopic evolution of supernova ejecta in the nebular phase have been presented by several groups (Fransson \& Chevalier 1989; Swartz 1991; Xu \& McCray 1991; Kozma \& Fransson 1992; Li \& McCray 1995). An updated review paper is given by McCray (1993). The basic mechanism involves energy deposition by gamma rays from radioactive materials $\left({ }^{56,57} \mathrm{Co}\right.$ and ${ }^{44} \mathrm{Ti}$ ) in the supernova ejecta. The fast electrons produce heating, excitation, and ionization. Five years after the supernova explosion, excitation and ionization dominate the energetics, while heating becomes unimportant due to the low ionization fraction of the ejecta. For most ions, recombination time scales in the SN 1987A ejecta at late times may exceed the dynamical and radioactive decay time scale and the ionization stage of the ejecta will freeze out (Clayton, Leising, \& The 1992; Fransson \& Kozma 1993). As a result, emission lines from radiative recombination remain strong long after the supernova outburst. Late time observations of SN 1987A provide us with an excellent opportunity to test these theories, and to analyze the ejecta.

The purpose of this paper is to present the pre-COSTAR HST observations of SN 1987A, present plausible explanations of the physical processes, and to derive some properties of the underlying event. We discuss in $\S 2$ the observations and data reduction. The results from this study are given in $\S 3$, where we discuss emission lines arising from hydrogen, oxygen, magnesium and iron, and the properties of the dust in SN 1987A. We give conclusions from this study in $\S 4$.

\section{Observations and Data Reduction}

The spectra of SN 1987A were acquired using the HST as part of the Supernova INtensive Study. Observations with the FOS were obtained on 1992 March 31, 1993 March 14, and 1993 April 1-6. Post-COSTAR observations were made on 1995 Jan. 7, and will be the subject of a subsequent paper. In addition to these spectroscopic observations, there are also WFPC images taken as part of the same project. The direct images have been discussed by Plait et al. (1994) and by Pun et al. (1996). This paper concentrates on the two pre-COSTAR spectra.

The data were reduced using the standard HST post-processing software. The pipeline-calibrated data were reduced with the most current calibration reference files. Table 1 contains the summary of the observations including dataset name, date of 
observation, detector, grating, and exposure time. Table 2 contains the observational parameters including wavelength coverage, resolution, and dispersion. All the data were taken through the $0^{\prime \prime} .25 \times 2^{\prime \prime} .0$ slit. The slit was located to observe both the broad lines from the debris and narrow lines from the circumstellar ring simultaneously. Figure 1 shows the slit orientation and position for each set of observations. Both the 1992 March 31 and 1993 March 14 observations were centered on the debris. Figure 1 was created from a FOC observation of SN 1987A on 1992 April 12. The 1993 April 1-6 observations were not centered on the target, our best estimate is that the slit was positioned $\sim 0^{\prime \prime} .3$ east of the debris. These miscentered observations show spectral features of the circumstellar ring, but no features from the debris. A new acquisition strategy has since been implemented to insure the aperture is well centered on SN 1987A.

At the off axis location of the FOS, the width (FWZI) of the Point Spread Function (PSF) of the optical system is about 2-3". Figure 1 shows that the light from star 3 ( $1^{\prime \prime} .3$ away) overlaps the position of the FOS aperture. Since star 3 is a Be star (Wang et al.. 1992; Walborn et al., 1993), the contamination is most serious in the UV. The amount of star 3 light in the supernova spectra can be estimated by two methods. First, the FOC images can be used to determine how much of the star 3 light enters the FOS aperture. Second, the FOS observations from 1995 Jan., during the COSTAR era, can be used as a guide to adjust the pre-COSTAR data. A correction can be calculated by measuring the difference in the flux level between the emission lines of the pre-COSTAR and the post-COSTAR spectra. Both methods give a consistent correction to the data. The contamination of the observed spectra by star 3 varies with wavelength, and ranges from as large as $75 \%$ at $2000 \AA$ to $17 \%$ at $4000 \AA$. At wavelengths longer than $5000 \AA$, this contamination is below $1 \%$. The observations made with the blue side of G130H FOS are completely dominated by Star 3 light. No emission features were detected from either the debris or the circumstellar ring.

\section{Results}

The final flux calibrated spectra are corrected for interstellar extinction assuming $E(B-V)=0.2 \mathrm{mag}$ (Panagia et al. 1987) and $A_{v}=0.62$. The spectra are shown in Figure 2 with the suggested line identifications. The original spectra consist of both narrow forbidden lines typical of HII regions and broad lines of FWHM greater than $2000 \mathrm{~km} \mathrm{~s}^{-1}$. The narrow lines were first observed with the IUE (Fransson et al. 1989) in the ultraviolet about 80 days after the supernova explosion, and from the ground (Wampler \& Richichi 1989 ) in the optical about 300 days after the explosion. These lines are believed to be due 
to the circumstellar material in the immediate SN 1987A environment, presumably material lost by the progenitor's stellar winds. The broad lines are from the supernova debris itself, and are the main topic of the current study. All the narrow lines have been removed from the spectra in Figure 2, but form the subject of a future paper (Panagia et al. 1995).

A complete list of the emission lines from the debris is given in Table 3. The wavelengths given in the table were approximate meassured values of the line peaks. The line strengths were measured by fitting a simple Gaussian profile with a linear background. There are three main difficulties in measuring the line strengths. First, there are the narrow emission features from the circumstellar ring blended with the broader debris lines. These emission features were fit with a single Gaussian profile with a linear background and subtracted. Secondly, the debris emission features are not exactly Gaussian, as discussed in $§ 4$. Line strengths obtained by simply integrating above a continuum are accurate to within this uncertainty, and therefore only values from Gaussian fits are tabulated. Third, the continuum level is often difficult to measure as many of the lines are blended. The estimate of the uncertainty of the line strength is 10-20 percent including also the uncertainties in the calibration of the absolute flux level. However, the relative strengths of the lines are more reliable, and are accurate to about 5 percent. The observed $\mathrm{H} \alpha$ fluxes are $1.66 \times 10^{-13} \mathrm{erg} \mathrm{cm}^{-2} \mathrm{~s}^{-1} \AA^{-1}$ and $1.17 \times 10^{-13} \mathrm{erg} \mathrm{cm}^{-2} \mathrm{~s}^{-1} \AA^{-1}$ for the 1992 and 1993 spectra, respectively. The dereddened $\mathrm{H} \alpha$ fluxes for the 1992 and 1993 spectra are $2.62 \times 10^{-13} \mathrm{erg} \mathrm{cm}^{-2} \mathrm{~s}^{-1} \AA^{-1}$ and $1.84 \times 10^{-13} \mathrm{erg} \mathrm{cm}^{-2} \mathrm{~s}^{-1} \AA^{-1}$, respectively.

Most of the lines which appeared in earlier nebular phase spectra, such as those identified by Phillips \& Williams (1991), and Kirshner et al. (1987) are still detectable. The strongest lines are $\mathrm{H} \alpha, \mathrm{H} \beta$, and [O I] $\lambda \lambda 6300,6364$ in the optical, and the resonance Mg I $\lambda 2852$, Mg II $\lambda \lambda 2795,2802$ lines discussed in detail by Pun et al. (1995) in the ultraviolet. A remarkable feature shown by the current data is that some of the iron lines, such as the [Fe II] $\lambda 7155$ line, which were strong in the early spectra have now disappeared. The temperature of the supernova ejecta is too low, and excitations by thermal electrons are no longer important for optical emission lines like [Fe II] $\lambda 7155$ at this epoch. As a consequence, most of the thermally excited emission lines should appear in the infrared where thermal excitations are still important. This behavior is related to the so-called infrared catastrophe as studied in detail by Fransson \& Chevalier (1989).

An interesting problem is that there are still several strong lines without secure identification, notably the emission features at wavelength $3630 \AA$ and $3727 \AA$. These lines will be discussed in more detail in the subsequent sections of this paper. We show that these are likely to be the Balmer continuum at low temperature and [O II] produced by ionization of neutral oxygen to excited states of $\mathrm{O}^{+}$. 


\subsection{Hydrogen Emission}

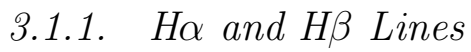

Hydrogen Balmer lines remain the strongest optical lines. The profiles of $\mathrm{H} \alpha$ and $\mathrm{H} \beta$ lines show an asymmetric redshifted tail out to $10000 \mathrm{~km} \mathrm{~s}^{-1}$ for $\mathrm{H} \alpha$ and $7000 \mathrm{~km} \mathrm{~s}^{-1}$ for $\mathrm{H} \beta$ beyond which the line profile is corrupted by an iron line. The profiles are shown in Figure 3 for the two epochs of observation. Both the $\mathrm{H} \alpha$ and $\mathrm{H} \beta$ profiles show the same asymmetry, so this is an intrinsic feature of the hydrogen, not a blending with other lines.

The asymmetric component in the line profile can be extracted by flipping the blue side of the $\mathrm{H} \alpha$ profile about the line peak and subtracting it from the red wing of the line profile. This results in a bump as broad as $8000 \mathrm{~km} \mathrm{~s}^{-1}$ (FWZI) centered at a redshift about 4000 $\mathrm{km} \mathrm{s}^{-1}$. The central velocity of this asymmetric component is in remarkable agreement with the analysis of the redshifted lines observed between day 200 and day 500 (Spyromilio et al. 1990), and the red emission satellite of the hydrogen lines between day 20 and day 100, the asymmetry noted by Hanuschik \& Dachs (1987), Larsonet et al. (1987), Phillips \& Heathecote (1989) and Spyromilio et al. (1990). Chugai (1991a, 1991b) suggested that this asymmetric component is due to an asymmetric distribution of radioactive clumps in the SN 1987A ejecta.

\subsubsection{Hydrogen Balmer Continuum}

Another interesting feature is at wavelength $3630 \AA$. While this appears to be an emission line with width comparable to other lines, we identify it as hydrogen Balmer continuum emission, based on the argument developed by Williams (1982) in his spectroscopic analysis of the shells around novae. The nova shells Williams analyzed are quite cold, with electron temperature of only about $800 \mathrm{~K}$. They emit unusual spectra in which recombination lines, including some forbidden lines formed by recombination, are quite strong. Because the emission coefficient for the Balmer continuum varies as $j_{\nu} \sim \exp \left[h\left(\nu_{\mathrm{o}}-\nu\right) / k T_{e}\right]$, where $\nu_{\mathrm{o}}$ is the Balmer ionization frequency, the Balmer continuum profile drops very sharply at frequencies higher than $\nu_{\mathrm{o}}$ when the kinetic temperature of the free electrons is very low, producing a narrow emission feature in the spectrum.

This identification of the Balmer continuum profile provides us with a powerful method for estimating the electron temperature in the ejecta. In order to do so, we have constructed a simple model which assumes that (a) there is a uniform distribution of hydrogen atoms 
inside a sphere of velocity smaller than $3000 \mathrm{~km} \mathrm{~s}^{-1}$; (b) the temperature is constant across the entire nebula; (c) the ejecta are optically thin to Balmer continuum photons. Assumption (a) is required mainly because of the lack of knowledge of the density structure of the debris; however, it is perhaps not very far from reality considering the complex chemical mixing processes indicated by various observations and models (see, McCray, 1993 for a review of this topic). Assumption (b) implies that the temperature to be deduced is only an average value. Assumption (c) can be justified because the ejecta are now much more extended than in early phases of evolution, and are now transparent to Balmer continuum photons (e.g., McCray 1993).

The blue side of the Balmer continuum profile is temperature sensitive, but is insensitive to the density structure. On the other hand, the redshifted portion of the Balmer continuum is determined primarily by the density structure of the ejecta, in which different velocities give different weights which alter the Doppler smearing of the Balmer edge. By matching both the blueshifted and redshifted parts of the profile, we are able to deduce the temperature of the ejecta quite accurately. The model profiles are plotted together with the observed line profiles in Figure 4. The corresponding best values of the electron temperature are $500 \mathrm{~K}$ on 1992 March 31 and $400 \mathrm{~K}$ on 1993 March 14. The uncertainties in these estimates are less than $100 \mathrm{~K}$, and they result mainly from uncertainties in setting the underlying continuum level below the Balmer feature. The temperatures deduced are reliable for a wide range of possible density structures. Numerical experiments show that a uniform density sphere with radius at velocities ranging from 2500 to $4000 \mathrm{~km} \mathrm{~s}^{-1}$ provides satisfactory fits to both the 1992 March and 1993 March observations and yields practically the same electron temperatures for each epoch.

As an additional test of the identification of the $3630 \AA$, feature with the Balmer continuum profile, we have estimated the electron densities required to produce the observed total flux in the Balmer continuum. Adopting the same model parameters as used in deducing the line profiles, the average electron densities required are found to be $3.2 \times 10^{3}\left(v / 3000 \mathrm{~km} \mathrm{~s}^{-1}\right)^{-3 / 2} \mathrm{~cm}^{-3}$ and $2.5 \times 10^{3}\left(v / 3000 \mathrm{~km} \mathrm{~s}^{-1}\right)^{-3 / 2} \mathrm{~cm}^{-3}$ for the 1992 and 1993 data, respectively. For comparison, we can also derive electron densities using the $\mathrm{H} \alpha$ line. Assuming that recombination of thermal electrons is the only process responsible for the $\mathrm{H} \alpha$ line, the electron density for both epochs is found to be $6.3 \times 10^{3}\left(v / 3000 \mathrm{~km} \mathrm{~s}^{-1}\right)^{-3 / 2} \mathrm{~cm}^{-3}$ at day 1862 . This is two to three times larger than the densities derived for the Balmer continuum. However, considering both the uncertainties in the absolute calibrations of the spectra and the over-simplified nature of the model, this agreement is satisfactory and adds to the evidence that our identification is correct. The derived electron density implies that the hydrogen-rich region is mostly neutral with ionization fraction around $1.2 \times 10^{-4}\left(\mathrm{M}_{\mathrm{H}} / 10 \mathrm{M}_{\odot}\right)^{-1}$. 
Chugai et al. (1993) reported identification of the Paschen continuum in the infrared, and used the Paschen continuum to determine the temperature of the hydrogen emitting region. The redshifted part of the Paschen continuum is corrupted by some unidentified features, and the temperature determination is less accurate than that derived here from the Balmer continuum. Nonetheless, the temperatures obtained from the Paschen continuum are consistent with what we have derived in this study.

\subsection{Oxygen Lines}

\subsection{1. $[O \quad \mathrm{I}] \lambda \lambda 6300,6364$ Doublet}

Chugai (1988) noted that in the case of supernova explosions, forbidden lines such as [O I] $\lambda \lambda 6300,6364$ are optically thick for about one year after the explosion, due mainly to the high densities encountered. The doublet ratio $R=F([\mathrm{OI}] \lambda 6300) / F([\mathrm{OI}] \lambda 6364)$ will be smaller than its optically thin limit of 3 . The ratio $R$ can be used to estimate the average density of the oxygen emitting region. Time evolution of the line ratio $R$ was indeed observed by Phillips \& Williams (1991) who found $R \approx 1$ at about 100 days after explosion increasing to about 2.6 at about 500 days after the explosion. Several authors have used this method to calculate the oxygen density in the SN 1987A ejecta (Spyromilio \& Pinto 1991; Li \& McCray 1992). The oxygen density derived from this method is $n_{O I}=1.3 \times 10^{9} t_{y}^{-3} \mathrm{~cm}^{-3}$. This is too large if all the oxygen is distributed uniformly, and suggests that most of the oxygen is in dense clumps with a volume filling factor $f_{\mathrm{O}} \approx 0.09 M_{\mathrm{O}} / M_{\odot}$. Five years after the outburst, the density of the ejecta has greatly decreased, and the $\left[\begin{array}{ll}\mathrm{O} & \mathrm{I}\end{array}\right] \lambda \lambda 6300,6364$ doublet should turn optically thin with a line ratio $R=3$. However, the simple two-Gaussian profile fits yield a ratio $R=1.9 \pm 0.1$ for both the 1992 and 1993 observations. This ratio is significantly different from the optically thin

limit of 3, and is even smaller than the previously reported ratio of 2.6 on day 500 (Phillips \& Williams 1991).

If we still apply Chugai's method for density determination, we would arrive at an oxygen density of $4.1 \times 10^{8} \mathrm{~cm}^{-3}$ on day 1862 , and $3.7 \times 10^{8} \mathrm{~cm}^{-3}$ on day 2210 . If the oxygen mass is of the order of $1-2 \mathrm{M}_{\odot}$, and the majority of the oxygen mass is distributed inside a shell with maximum velocity of $1700 \mathrm{~km} \mathrm{~s}^{-1}$ (as used by Li \& McCray 1992), a volume filling factor of $\sim 2 \times 10^{-3}$ can be derived. This is about two orders of magnitude smaller than the value deduced by Li \& McCray (1992) using the same lines but for early observations. The latter authors found a volume filling factor of about 0.12 by modelling observations prior to day 500. Such a change could occur if the majority of the oxygen material is not emitting efficiently at late times, and only the densest parts of the ejecta 
are responsible for the observed line fluxes. In such a scenario, the early observations show the less dense part of the ejecta, while the later observations show the denser clumps of the ejecta which occupy less volume. This possibility, however, is unlikely as judged by the physical processes responsible for the line emission. The SN 1987A ejecta are expanding homologously and the density should drop as $t_{y}^{-3}$. At such late stages of the supernova ejecta, as we have derived above from the Balmer continuum, the electron temperature is as low as several hundred degrees Kelvin. This means that thermal excitations are negligible in powering the emission lines, and the $\left[\begin{array}{ll}\mathrm{O} & \mathrm{I}\end{array}\right] \lambda \lambda 6300,6364$ doublet photons are produced purely by non-thermal excitations by fast electrons from gamma ray deposition in the ejecta. Since the ejecta are now optically thin to gamma rays, the energy deposited per atomic oxygen is nearly independent of local oxygen density. We would then expect that all oxygen atoms are excited equally; thus, it does not seem likely that only the densest part in the ejecta contributes to the [O $\mathrm{I}] \lambda \lambda 6300,6364$ emission. Another possibility is that the oxygen is becoming more clumpy with time, but this would require strong deviations from homologous expansion of the ejecta. No physical model leads naturally to this situation.

Blending with other lines may also be possible. Lines that are close to [OI ] $\lambda 6364$ include those from Fe I multiplet 13, forbidden transitions of Fe I mulitplet F17, FeII multiplet 40, and Ti I multiplet 1 . Line blending may affect the measured line ratio, but quantitative estimates of the effect are difficult. Here we restrict our analysis to contributions by a single third emission line having the same velocity structure as $[\mathrm{O}$ I] $\lambda \lambda 6300,6364$. The observed line profiles are modelled by minimizing $\chi^{2}$ fits of three Gaussians. We assume: (a) all three Gaussians have the same FWHM; (b) the contributions from [O I] $\lambda \lambda 6300,6364$ are given by two Gaussians with intensity ratio of 3/1 of which the weaker component is redshifted by $3047.6 \mathrm{~km} \mathrm{~s}^{-1}(64 \AA)$ with respect to the blue component. The approximate continuum level is simultaneously fitted by a linear function. Figure 5a shows the results of such experiments. The goodness of the fit is considerably improved compared with the two-Gaussian fit described above. The strengths of the postulated third component are $14.4 \%$ and $27.3 \%$ of the total line fluxes in the 1992 and 1993 spectra, respectively. The absolute strength and the profile of this component did not change during the two observations. The increase in its relative contribution to the total line intensity was due to a decrease in the total line intensity. The centroid of the third Gaussian is redshifted by $2500 \pm 73 \mathrm{~km} \mathrm{~s}^{-1}(52 \pm 1.5 \AA)$ with respect to the [O I] $\lambda 6300$ line. The iron line closest in wavelength is from Fe I $\lambda 6355.6$ (multiplet 13). However, Fe I $\lambda 6355.6$ should be blended with other Fe I multiplet 13 lines at wavelength $6402.1 \AA$, $6360.5 \AA$, and $6282.4 \AA$. Because the permitted Fe I lines can be optically thick at the time of the observations, these lines may be as strong as Fe I $\lambda 6355.6$. The overall contribution from the Fe I multiplet 13 should thus have a profile very different from that of a single 
line. The lines of Fe II multiplet 40, and Ti I multiplet 1, although having acceptable wavelengths, may have the same difficulties in explaining the observed line ratio. An alternative explanation of the abnormal line ratio is that the [Fe II] $\lambda \lambda 6340,6444$ (15F) line is strong in SN 1987A (Spyromilio, 1995, private comunication). The problem with this possibility is that the [Fe II] $\lambda 6444$ line is usually stronger than the [Fe II] $\lambda 6340$, and it is too far to the red side of the [O I] $\lambda 6364$ line to fit the observations. Unless a reliable model of the iron emission process is available, it is hard to analyse the iron lines quantitively. Nevertheless, it seems difficult to account for the observed [O $\mathrm{I}] \quad \lambda \lambda 6300,6364$ line ratio by contamination with a third component.

An alternative explanation is that the ratio deduced by line profile fitting is an artifact of the technique. In fact, by assuming different line profiles, one gets quite different values of the line ratios with satisfactory matches to the observed line profiles. A unique solution of the line ratio $R$ is never guaranteed. To be more specific, the problem of the doublet decomposition is described by the following simple equation

$$
F(\lambda)=A_{1}(\lambda)+A_{2}\left(\lambda+\Delta \lambda_{0}\right) / R,
$$

where $F$ is the flux of the $\left[\begin{array}{ll}\mathrm{O} & \mathrm{I}\end{array}\right] \lambda \lambda 6300,6364$ doublet, $\lambda$ is the wavelength, $\Delta \lambda_{0}$ is the wavelength difference of the two lines which is equal to 64 Åin the present case, $A_{1}$ and $A_{2}$ are the line profiles for the $6300 \AA$ and $6364 \AA$ lines, respectively. We will assume that the [O I] $\lambda 6300$ and [O I] $\lambda 6364$ lines have the same line profile; $A_{1}$ and $A_{2}$ are then identical.

Instead of using Gaussian profiles to calculate the ratio $R$, we reverse the process by fixing the ratio $R$ and calculating the resulting line profile $A$. This avoids the ambiguities of unknown line profiles, and produce a clearer physical picture. In addition, the solution is mathematically unique for each value of $R$. We show in Figure $5 \mathrm{~b}$ the decomposed profiles of the [O I] $\lambda 6300$ and [O I] $\lambda 6364$ lines together with the observed profile of the $\mathrm{Mg} \mathrm{I}]$ $\lambda 4571$ line for comparison, assuming a line ratio $R=3$ for the [OI] doublet. It is surprising that such a simple technique yields [O I] $\lambda 6300$ line profiles that are strikingly similar to those of the Mg I] $\lambda 4571$ line. This agreement suggests that the decomposed profiles are adequate representations of each individual component of the [O $\mathrm{I}] \lambda \lambda \lambda 6300,6364$ doublet, and argues strongly that the line ratio is in good agreement with the theoretical value expected in the optically thin limit, $R=3$. Least $\chi^{2}$ fit of the doublet ratio using the $\mathrm{Mg}$ I] $\lambda 4571$ line as a template yields numbers of $3.1 \pm 0.3$ for the two epochs of observations. The uncertainties arise largely from the difficulties in defining the continuum level for the lines.

The present analysis shows that the line ratio depends sensitively on the assumed line profile. It raises doubts concerning the early [O $\mathrm{I}] \lambda \lambda 6300,6364$ doublet ratios derived by line profile fitting (Phillips \& Williams 1991; Spyromilio \& Pinto 1992). We believe that 
a more careful analysis of the early data, considering the approaches outlined above, is required before drawing quantitative conclusions about the oxygen density in the SN 1987A ejecta. A detailed comparison of the early optically thick oxygen profiles with the later optically thin profiles is of critical importance to establish a quantitative picture of the oxygen emitting region in the SN 1987A ejecta.

\subsection{2. $[O \mathrm{II}] \lambda \lambda 3726,3728$}

The prominent [O II] $\lambda \lambda 3726,3728$ line requires some remarks. Surprisingly, this line is one of the strongest in the SN 1987A spectra - in fact, second only to $\mathrm{H} \alpha$. As for the [O I] $\lambda \lambda 6300,6364$ lines, the temperature of the ejecta is too low for thermal electrons to excite the $[\mathrm{O}$ II $] \lambda \lambda 3726,3728$ doublet at the epochs of these observations, so only non-thermal excitations of the $\mathrm{O}^{+}$ions are important. The ionization fraction of the oxygen-rich region five years after explosion is less than 0.001 as shown by several calculations (Fransson \& Chevalier 1989; Xu \& McCray 1992; Kozma \& Fransson 1992); therefore, only a miniscule fraction of the oxygen is ionized. Detailed calculations which take into account non-thermal excitations show that the expected [O II] $\lambda \lambda 3726,3728$ doublet strength should be less than 10 percent of that of the [O I] $\lambda \lambda 6300,6364$ lines (Kozma \& Fransson 1992), while the observed line strength of [O II] $\lambda \lambda 3726,3728$ is considerably larger than that of $\left[\begin{array}{ll}\mathrm{O} & \mathrm{I}\end{array}\right]$ $\lambda \lambda 6300,6364$.

As discussed by Williams (1982) in his explanation of the unusual spectra of the shells of novae, another way to produce the [O II] $\lambda \lambda 3726,3728$ lines may be the recombination of $\mathrm{O}^{++}$ions. The [O II] $\lambda \lambda 3726,3728$ lines are the strongest optical recombination lines from $\mathrm{O}^{++}$. However, taking the effective recombination rates for the $[\mathrm{O}$ II] $\lambda \lambda 3726,3728$ doublet as given by Péquignot et al. (1991), it is easy to verify that recombinations produce an insignificant amount of [O II] $\lambda \lambda 3726,3728$ photons.

A more natural mechanism for powering the [O II] $\lambda \lambda 3726,3728$ doublet is ionization of atomic oxygen directly to excited states of $\mathrm{O}^{+}$in a single impact. Updated excitation and ionization cross sections for electron impact on atomic oxygen by Laher \& Gilmore (1989) include the cross sections of collisional ionization to excited states of $\mathrm{O}^{+}$. Their results show that for electrons with energy above several tens of electron volts, a significant fraction of the ionizations of the neutral oxygen end up with $\mathrm{O}^{+}$ions in their ${ }^{2} D^{0},{ }^{2} P^{0}$, and ${ }^{4} P$ states. For example, when the energy of the free electron is $50 \mathrm{eV}$, about $20.6 \%$ of the total ionized $\mathrm{O}^{+}$ions go to the ${ }^{2} D^{0}$ state, and $59.4 \%$ go to the ground state ${ }^{4} S^{0}$; at very high energies $(>1000 \mathrm{eV})$, the ionization to the ground state of the $\mathrm{O}^{+}$line is $36 \%$, to ${ }^{2} D^{0}$ is $30 \%$, to ${ }^{2} P^{0}$ is $17 \%$, and to ${ }^{4} P$ is $18 \%$. The fractions going into excited states are almost 
independent of the energy of the free electrons at energies above $1000 \mathrm{eV}$.

The spontaneous decay of the levels ${ }^{2} D^{0},{ }^{2} \mathrm{P}^{0}$, and ${ }^{4} \mathrm{P}$ of $\mathrm{O}^{+}$can be an efficient route to produce [O II] $\lambda \lambda 3726,3728$ and [O II] $\lambda \lambda 7319,7330$ photons. The only path for the decay of ${ }^{2} D^{0}$ is to the ground level with the emission of [O II] $\lambda \lambda 3726,3728$. The ${ }^{2} P^{0}$ level decays to the ground level producing [OII] $\lambda 2471$ or to the ${ }^{2} D^{0}$ level with the emission of $[\mathrm{OII}] \lambda 7330$, with a branching ratio of 0.3775 . For each ionization of neutral oxygen, the number of [O II] $\lambda \lambda 3726,3728$ photons produced is then

$$
\xi=r_{2} D^{0}+0.3775 r_{2} P^{0},
$$

where $\xi$ is the fraction of ionizations to the excited states of $\mathrm{O}^{+}$, and $r_{2} D^{0}$ and $r_{2} P^{0}$ are the fraction of ionizations to the states ${ }^{2} D^{0}$ and ${ }^{2} P^{0}$, respectively. Collisional de-excitation of the ${ }^{2} D^{0}$ level can be important, further reducing the line flux approximately by a factor of $\eta=1 /\left(1+N_{e} / N_{c r}\right)$, where the critical density $N_{c r}=3.6 \times 10^{3} \mathrm{~cm}^{-3}$ at a typical temperature of $500 \mathrm{~K}$ (Osterbrock 1989), and $N_{e}$ is the electron density. The ionization of the neutral oxygen per second per cubic centimeter is

$$
4 \pi \frac{J_{\gamma} \sigma_{\gamma, O I}}{\chi_{e f f, O I}} n_{O I},
$$

where $J_{\gamma}$ is the mean gamma ray intensity, and $\sigma_{\gamma, O I}=\kappa_{\gamma} A_{O I} m_{p}$ with the mass absorption coefficient $\kappa_{\gamma}=0.06 Z_{O I} / A_{O I} \mathrm{~cm}^{2} \mathrm{~g}^{-1}$. Here $Z_{O I}=8$ and $A_{O I}=16$ are the atomic number and mass of the element.

For ionization fraction $x_{e}<<1$, the gamma ray mean intensity is given by (Kozma \& Fransson 1992)

$$
J_{\gamma}=\frac{D_{\gamma} L_{\gamma}}{16 \pi^{2}(v t)^{2}}
$$

where $D_{\gamma}$ is a geometric factor of order unity, $v$ is the velocity of the ejecta, and the luminosity of the gamma rays $L_{\gamma}$ is given by

$$
\begin{aligned}
L_{\gamma}= & 9.1 \times 10^{41}\left(\frac{M\left({ }^{56} N i\right)}{0.07 \mathrm{M}_{\odot}}\right)\left(e^{-t / 111.3}+9.3 \times 10^{-4} e^{-t / 391.2}\right)+ \\
& 4.1 \times 10^{36}\left(\frac{M\left({ }^{44} \mathrm{Ti}\right)}{10^{-4} \mathrm{M}_{\odot}}\right) e^{-t / 28489.5} \mathrm{erg} \mathrm{s}^{-1},
\end{aligned}
$$

assuming $0.07 \mathrm{M}_{\odot}$ of ${ }^{56} \mathrm{Ni}$ and ${ }^{57} \mathrm{Ni} /{ }^{56} \mathrm{Ni}$ is 1.5 times the solar ${ }^{57} \mathrm{Fe} /{ }^{56} \mathrm{Fe}$ ratio, or $2.5 \times 10^{-3}$ $\mathrm{M}_{\odot}$. The [O II] $\lambda \lambda 3726,3728$ flux is then estimated to be

$$
\begin{aligned}
F_{[O I I]}= & 5.5 \times 10^{-10} \eta D_{\gamma}\left(\frac{L_{\gamma}}{9.1 \times 10^{41} \mathrm{erg} \mathrm{s}^{-1}}\right)\left(\frac{v}{2000 \mathrm{~km} \mathrm{~s}^{-1}}\right)^{-2}\left(\frac{t}{2000 \text { days }}\right)^{-2} \\
& \left(\frac{M_{\mathrm{O}}}{\mathrm{M}_{\odot}}\right)\left(\frac{D}{50 \mathrm{kpc}}\right)^{-2} \operatorname{erg~cm}^{-2} \mathrm{~s}^{-1}
\end{aligned}
$$


where $D$ is the distance to the LMC, and $t$ is in units of days, and we have taken $\chi_{e f f, O I}=26.7 \mathrm{eV}$.

As a further test of our line identification, we need to estimate the amount of oxygen mass required to produce the [O II] $\lambda \lambda 3726,3728$ doublet. The line fluxes after 1500 days are also very sensitive to the exact amount of energy input to the ejecta, a higher energy input can reduce the mass of oxygen required to reproduce the observed line flux. Woosley \& Hoffman (1991) argue that the ${ }^{44} \mathrm{Ti}$ mass could range from $10^{-6}$ to $10^{-4} \mathrm{M}_{\odot}$. In a more recent calculation, Timmes et al. (1995) found an upper limit of $\sim 1.5 \times 10^{-4} \mathrm{M}_{\odot}$ of ${ }^{44} \mathrm{Ti}$ that can be synthesized in a Type II supernova like SN 1987A. Late time observations show that although time dependent effects (Fransson \& Kozma 1993) can reduce the amount of energy sources required to fit the bolometric light curve, some additional energy source such as an accreting X-ray pulsar, and/or a pulsar are still necessary to fit the observations (Bouchet et al. 1993).

The observed [O II] $\lambda \lambda 3726,3728$ flux corrected by interstellar extinction on day 1862 is $8.6 \pm 1.6 \times 10^{-14} \mathrm{erg} \mathrm{cm}^{-2} \mathrm{~s}^{-1}$, and on day 2210 it is $7.1 \pm 1.4 \times 10^{-14} \mathrm{erg} \mathrm{cm}^{-2} \mathrm{~s}^{-1}$. These numbers are reasonably reproduced by assuming a ${ }^{44}$ Ti mass of $4 \times 10^{-4} \mathrm{M}_{\odot}$, and still keeping the mass of oxygen in a range that is consistent with that obtained by Fransson, Houck, \& Kozma (1994). We found from equations (7) and (8),

$$
\eta D_{\gamma} M_{\mathrm{O}}\left(v / 2000 k m s^{-1}\right)^{-2}=5.1 \mathrm{M}_{\odot}
$$

for the 1992 observation and

$$
\eta D_{\gamma} M_{\mathrm{O}}\left(v / 2000 \mathrm{kms}^{-1}\right)^{-2}=7.6 \mathrm{M}_{\odot}
$$

for the 1993 observation, respectively. In a simple type of analysis similar to the above, Fransson, Houck, \& Kozma (1994) derive a lower limit of $M_{\mathrm{O}} \geq 3\left(v / 1500 \mathrm{~km} \mathrm{~s}^{-1}\right)^{2} \mathrm{M}_{\odot}$. If we assume, as derived by Kozma \& Fransson (1992), that $D_{\gamma} \leq 3$, we then obtain, for typical velocities of the oxygen shell around $1500 \mathrm{~km} \mathrm{sec}^{-} 1$, a lower limit for the oxygen mass of $\eta M_{\mathrm{O}} \geq 0.96\left(v / 1500 \mathrm{~km} \mathrm{~s}^{-1}\right)^{2} \mathrm{M}_{\odot}$. The correction for collisional excitation requires knowledge of the electron density which depends further on the clumpiness and ionization fraction of the ejecta. To be consistent with Fransson, Houck, \& Kozma (1993), we require $\eta$ to be around 0.3 , which can be achieved if the electron density is about $8.4 \times 10^{3} \mathrm{~cm}^{-3}$. The corresponding ionization fraction is $2.8 \times 10^{-3} f_{\mathrm{O}}$ for $3 \mathrm{M}_{\odot}$ of oxygen distributed inside a sphere with maximum velocity $1500 \mathrm{~km} \mathrm{~s}^{-1}$, where $f_{\mathrm{O}}$ is the volume filling factor of the ejecta. It should be noted that the oxygen masses derived in the simplified approach of Fransson, Houck, \& Kozma (1994) are larger than the $1.2-1.5 \mathrm{M}_{\odot}$ derived from the $[\mathrm{O}$ I] $\lambda \lambda 6300,6364$ lines by Li \& McCray (1992) and Chugai (1994). In their more detailed models, a lower limit of oxygen mass of $1.5 \mathrm{M}_{\odot}$ was derived. The oxygen mass derived 
by Fransson, Houck, \& Kozma (1994) is also larger than or close to the upper limit of the $0.24-1.6 \mathrm{M}_{\odot}$ predicted by explosion models of the SN 1987A progenitor (Hashimoto, Nomoto, \& Shigeyama 1989; Woosley 1988; Thielemann, Nomoto, \& Hashimoto 1995). It remains to be seen if a revised analysis of both the line fluxes and profiles of the earlier $[\mathrm{O}$ I] $\lambda \lambda 6300,6364$ data will bring these numbers into agreement. However, it is encouraging that the order of oxygen mass required to produce the fluxes of the [O II] $\lambda \lambda 3726,3728$ line is correct even in this simple analysis.

The [O II] $\lambda \lambda 3726,3728$ line can also be used to put a limit on the electron density in the oxygen-rich region. One approach is to solve the ionization equilibrium time dependently, in a way similar to that for the $\left[\begin{array}{ll}\mathrm{O} & \mathrm{I}\end{array}\right] \lambda \lambda 6300,6364$ line described by Fransson, Houck, \& Kozma (1994). The line strengths and profiles set constraints on both the oxygen mass and its geometrical distribution. We outline here a simpler method for obtaining the electron density. Because of the freeze-out effect, the total number of recombinations per unit time will be larger than the total number of ionizations. An upper limit for the [O II] $\lambda \lambda 3726,3728$ luminosity is obtained by assuming that each ionization to the excited states of $\mathrm{O}^{+}$decays only via the radiative process:

$$
\xi h \nu \alpha_{\mathrm{O}^{+}} N_{\mathrm{OII}} N_{e} / f_{\mathrm{O}}^{2} V \geq L_{[\mathrm{OII}] 3727},
$$

where $\nu$ is the frequency of the transition, $\alpha_{\mathrm{O}^{+}}$the recombination coefficient of $\mathrm{O}^{+}$which equals $3.201 \times 10^{-13} T_{4}^{-0.688} /\left(1-0.0174 T_{4}^{1.707}\right)$ with $T_{4}$ being the temperature in units of $10^{4} \mathrm{~K}$ (Arnaud \& Rothenflug 1985), and V the volume of the emitting region. Taking the observed fluxes for $L_{[\mathrm{OII}] 3727}$, it is easy to derive from equation (10) that

$$
<N_{\mathrm{OII}} N_{e}>^{1 / 2} \geq 5400 f_{\mathrm{O}}^{-1}\left(\frac{v}{2000 \mathrm{~km} \mathrm{~s}^{-1}}\right)^{-3 / 2}\left(\frac{t}{2000 \text { days }}\right)^{-3 / 2} \mathrm{~cm}^{-3}
$$

If we assume that mixing in the ejecta is macroscopic, equation (11) then gives approximate values of the average electron density in oxygen-rich regions of the ejecta. The corresponding recombination time scale of the oxygen-rich regions is then about 849 days, which is longer than the radioactive time scale, but smaller than the dynamical time scale. This confirms that time dependent effects (Kozma \& Fransson 1993) are important at the epoch of these observations.

The most useful aspect of the identification of the [O II $] \lambda \lambda 3726,3728$ line is that it provides an interesting method to estimate the amount of energy powering the ejecta. According to the mechanism outlined above, the [O II] $\lambda \lambda 3726,3728$ line is powered mainly by ionization followed imediately by excitations; because the spontaneous decay time scale of the excited level is only a few hours, much shorter than the radioactive time scale or the 
dynamical time scale, the time-dependent effects as studied by Kozma \& Fransson (1993) will never be important for the late time evolution of the [O II] $\lambda \lambda 3726,3728$ lines. The [O II] $\lambda \lambda 3726,3728$ line strengths should therefore provide a reliable measure of the energy sources powering the late time emission from supernova ejecta which is immune to time dependent effects. Here we have derived an equivalent of about $4 \times 10^{-4} \mathrm{M}_{\odot}$ of ${ }^{44} \mathrm{Ti}$ to power the late time spectra. The method may be complicated by blending with iron lines near $3727 \AA$ (Chevalier 1995, private comunications), in which case, it will provide an upper limit to the total amount of the underlying energy sources.

\subsection{Magnesium}

There are four magnesium lines observed in the spectrum. In addition to the semi-forbidden Mg I] $\lambda 4571$ line in the optical, the resonance lines Mg I $\lambda 2852$ and $\mathrm{Mg}$ II $\lambda \lambda 2795,2802$ are the strongest emission features in the ultraviolet. The most remarkable feature of the magnesium lines is the apparent difference of the line width in the ultraviolet and in the optical. Though it seems that all the lines show similar extended redshifted tails, the overall line profiles are much broader in the ultraviolet than in the optical. Notably, the FWHM of the Mg I $\lambda 2852$ line is more than twice the FWHM of the Mg I] $\lambda 4571$ line, and is broader than all of the optical lines. Because the Mg II $\lambda \lambda 2795,2802$ lines are blended, and its red wing blended with the Mg I $\lambda 2852$ line, it is impossible to deblend the lines and estimate their FWHM accurately. It is, however, very likely that the widths of the Mg II $\lambda \lambda 2795,2802$ lines are comparable with that of the Mg I $\lambda 2852$. The line profiles at the rest wavelength of Mg I $\lambda 2852$ at different epochs are shown in Figure 6, together with the $\mathrm{H} \alpha$ lines for comparison.

The difference in line width offers a straightforward explanation for the size difference of the SN 1987A ejecta in the optical and UV, as measured from the HST direct images obtained with FOC. Jakobson et al. (1994) showed that the SN 1987A ejecta are resolved by the HST. The measured size in the ultraviolet is twice that in the optical. Since the strongest optical lines are from decays of metastable levels which are optically thin, and the central wavelength for the UV image is at $2700 \AA$ which is close to the resonance lines of magnesium in the ultraviolet, the observed differences in the emission line width are clearly due to the fact that the UV lines are formed at higher velocity further out in the ejecta. This gives a natural explanation of the observed size for the ejecta. A detailed analysis of the ultraviolet and optical images is an interesting problem which we will defer to a separate study. 


\subsection{Iron Lines and Line Blending}

Numerous iron lines are present in the late time spectra of SN 1987A, although reliable identifications of these lines are difficult because the widths of the lines are so large that all the iron lines are strongly blended. However, in the ultraviolet, the Fe II emission lines from multiplet UV 1 and UV 2 are conspicuously strong. Iron probably makes a major contribution to the diffuse emission complex at wavelengths from $3000 \AA$ to $3850 \AA$; for instance, the Fe II optical multiplets $1,2,3,4,5,6,7,16$, and 29 can be the major contributors. Fe I lines from optical multiplets 4,5,6,20,21,23,24, and 25 may also be significant in SN 1987A; they are observed to be strong in the wavelength range from $3500 \AA$ to $3900 \AA$ in the narrow line quasar PHL 1092 (Bergeron \& Kunth 1980) and in the Seyfert galaxy IZwl (Oke \& Lauer 1979). Note that the Balmer continuum and the [OII] $\lambda 3727 \AA$ line lie just above this diffuse emission complex. The iron lines may affect the line profiles and intensities of the Balmer continuum and of [O II] $\lambda \lambda 3726,3728$. Quantitative estimates of this effect requires rigorous models of the iron emission which are difficult and uncertain. In our analysis of the Balmer continuum (§3.1.2) and the [O II] $\lambda \lambda 3726,3728$ line (§3.2.2), we have assumed that the iron lines from $3500 \AA$ to $3900 \AA$ are strongly blended and can be approximated by a smooth curve. This approximation is perhaps not too far from reality considering the fact that most of the Fe I and Fe II lines may be optically thick and severely blended. The emission feature from 4180 to $4515 \AA$ shows three distinct peaks. Emission lines in the same wavelength range exist also in IZwl and are identified with [Fe II] lines (Oke \& Lauer 1979). Some major contributors to these lines are [Fe II] $\lambda 4244 \AA$ (21 F), [Fe II] $\lambda 4287 \AA(7 F),[F e$ II] $\lambda 4320 \AA(21 \mathrm{~F})$, and [Fe II] $\lambda 4458 \AA(6 \mathrm{~F})$.

The profile of Mg I $\lambda 2852$ may also be affected by iron lines. However, the HST direct images show strong evidence that the size of the ejecta in the UV filter F275W is larger than that in the optical (§3.3; Jakobson et al. 1994). The Mg II $\lambda \lambda 2795,2802$ and Mg I $\lambda 2852$ lines contribute about $35 \%$ to the total integrated light in the F275W filter. The ejecta size in the direct UV images can be understood with an intrinsically broader profile of the lines without strong iron contamination.

\subsection{Asymmetries}

The highest detectable expansion velocities are given in Table 4 for the strongest lines. They are all measured on the redshifted side of the profiles. The lines that show the fastest expansion are $\mathrm{H} \alpha$ and $\mathrm{Mg}$ I $\lambda 2852$ lines, with velocities up to $10,000 \mathrm{~km} \mathrm{~s}^{-1}$. Such a high velocity for Mg I $\lambda 2852$ indicates clearly that a significant amount of the line flux is produced in the receding side of the hydrogen envelope of the ejecta. 
Comparisons between the line profiles of $\mathrm{H} \alpha, \mathrm{H} \beta, \mathrm{Mg} \mathrm{I}] \lambda 4571$, [O I I $\lambda \lambda 6300,6364$, and [O II] $\lambda \lambda 3726,3728$ show strong similarities. They are all blueshifted by a similar amount and exhibit extended redshifted wings. The blueshifts of the line peaks with respect to the narrow nebular lines vary from 300 to $500 \mathrm{~km} \mathrm{~s}^{-1}$. Considering the uncertainties in the measurements and the lack of clear measurable peaks for many lines, these numbers are about the same as those reported earlier from ground-based studies which show that the peaks are blueshifted by about 800 and $500 \mathrm{~km} \mathrm{~s}^{-1}$ for Mg I] $\lambda 4571$ and [OI]6300 (Bouchet et al. 1993) on around day 2000.

It has been convincingly shown that the blueshift of the emission lines is due to dust formation in the SN 1987A ejecta (Lucy et al. 1989). Lucy et al. (1991) show evidences of a two component structure of the dust, diffuse small grains which produce the selective extinction at short wavelength, and very dense dust clumps which give rise to the wavelength-independent effect. By the time of these observations, the ejecta had expanded several times and its density was so low that the diffuse component as studied by Lucy et al. $(1989 ; 1991)$ were optically thin. Applying the models of the line profiles of Lucy et al. (1989), we find that the optical depth required to account for the blueshifts in the current data is practically the same as in early measurements by Lucy et al. (1989; 1991). This property for the extinction can only be explained if most of the dust that condensed in the SN 1987A ejecta is distributed in dense clumps whose optical depth remains far above unity even at this late stage of evolution. Assuming that the dust clumps expand homologously, the optical depth then scales as $\sim t^{-2}$, and a lower limit for the optical depth of each individual dust clump on day 500 after explosion must be above 16. By requiring the dust blobs to be optically thick, we can derive a lower limit to the total mass of dust in the supernova ejecta as

$$
M_{\text {dust }} \geq 1.78 \times 10^{-5} \mathrm{M}_{\odot}\left(\frac{\tau}{0.4}\right)\left(\frac{\tau_{b}}{1}\right)\left(\frac{a}{0.1 \mu \mathrm{m}}\right)\left(\frac{v}{2000 \mathrm{~km} \mathrm{~s}^{-1}}\right)^{2}\left(\frac{\rho_{d}}{1 \mathrm{~g} \mathrm{~cm}^{-3}}\right),
$$

where $\tau$ is the effective optical depth of the dust blobs, $\tau_{b}$ is the optical depth of each individual dust blob, $a$ is the radius of the dust particles, and $\rho_{d}$ is the density of the dust grains.

Inspection of Figure 5a, b and 6 showed a weak but definite wavelength-dependent effect of the extinction, especially for the lines Mg I] $4571 \AA$, [O I] $6300 \AA$, and $\mathrm{H} \alpha$. In their analysis of observations collected at around day 550, Lucy et al. (1991) attributed this wavelength dependence as produced by the selective extinction of the duffuse dust component. At around day 2000, this diffuse component should have become optically thin and ineffective in affecting the line profiles. This may imply that dust formation continued even at the late stage, or that the dense dust clumps were only partially optically thick at around day 2000 . 
The origin of the red wing in each line profile is most likely to be the relic of the asymmetries observed in a much earlier phase, as shown by Phillips \& Heathecote (1989) and Hanuschik \& Dachs (1987) for the H $\alpha$ line and most clearly by Larson et al. (1987) for $\mathrm{P} \alpha$. Because the supernova is now powered mainly by the decay of ${ }^{57} \mathrm{Co}$ and ${ }^{44} \mathrm{Ti}$, it thus seems that ${ }^{57} \mathrm{Co}$ and ${ }^{44} \mathrm{Ti}$ are also asymmetrically distributed, in a way quite similar to that of ${ }^{56} \mathrm{Co}$.

\section{Conclusions}

We have analyzed the HST spectra of the ejecta of SN 1987A. The optical spectra of SN 1987A resemble strongly those of novae many decades after explosion (Williams 1992). This should not be surprising considering the fact that the temperatures of both the SN 1987A ejecta and the nova shells are comparable. The hydrogen Balmer continuum yields accurate estimates of electron temperature in the SN 1987A ejecta. The derived temperatures of the hydrogen emitting region are $500 \mathrm{~K}$ and $400 \mathrm{~K}$ on day 1862 and 2210, respectively. Such low temperatures are consistent with model calculations of Kozma \& Fransson (1992) and Fransson \& Kozma (1994).

The $\left[\begin{array}{ll}\mathrm{O} & \mathrm{I}\end{array}\right] \lambda \lambda 6300,6364$ doublet is decomposed into separate [OI] $\lambda 6300 \AA$ and $6364 \AA$ lines assuming they have an identical profile and an intensity ratio of $3: 1$. The decomposed [OI] profile is broadly consistent with the profiles of other lines such as $\mathrm{Mg}$ I] $\lambda 4571,[\mathrm{O} \mathrm{II}] \lambda \lambda 3726,3728$, and even the hydrogen Balmer lines. The line ratios $R=F([\mathrm{OI}] 6300) / F([\mathrm{OI}] 6364)$ cannot be estimated accurately without knowing the true $[\mathrm{OI}] \lambda 6300$ line profile. Early model calculations of the oxygen mass which used the doublet ratio $R$ derived on the basis of line profile fitting or line peak measurements are subject to this uncertainty. A robust analysis of the [ $\left[\begin{array}{ll}\mathrm{O} & \mathrm{I}\end{array}\right] \lambda \lambda 6300,6364$ doublet using the mechanism proposed by Chugai $(1988 ; 1994)$ can only be obtained by data analysis which makes a careful assessment of the line strengths without assuming a particular shape for the line profile. This needs to be applied uniformly to both early and late data.

We found that the $[\mathrm{O}$ II $] \lambda \lambda 3726,3728$ line has become surprisingly strong in the SN 1987A ejecta. The [O II] $\lambda \lambda 3726,3728$ doublet strength is modeled by considering collisional ionizations of neutral oxygen directly to excited states of $\mathrm{O}^{+}$. We note that the [O II] $\lambda \lambda 3726,3728$ line potentially gives an estimate of the oxygen mass. Our simple analysis sets a lower limit for the SN 1987A oxygen mass as shown by equation (10). The mechanism responsible for the [O II] $\lambda \lambda 3726,3728$ doublet is not unique to SN 1987A, but may be applicable to other supernovae as well. Moreover, the mechanism is not unique to atomic oxygen but may also apply to other abundant elements such as $\mathrm{C}$ and Ne. If 
this interpretation is correct, the [O II] $\lambda \lambda 3726,3728$ doublet will to be an interesting measure of the energy sources powering late time emission of the supernova, independent of time-dependent recombination.

The Mg I $\lambda 2852$ and Mg II $\lambda \lambda 2795,2802$ lines in the ultraviolet form at high velocity and large radius and thus provide a straightforward explanation for the size difference of the SN 1987A ejecta in the ultraviolet and in the optical as deduced from the HST direct images (Jakobson et al. 1992, 1994).

The ensemble of optical emission lines is blueshifted by an amount comparable to those reported earlier (Lucy et al. 1989, 1991), which requires continued high optical depth in dust from day 500 to 2000. A lower limit to the dust mass in the SN 1987A ejecta was derived as shown in equation (12). The line profiles are asymmetric; red shifted tails are observed for all the lines, up to $10000 \mathrm{~km} \mathrm{~s}^{-1}$ for $\mathrm{H} \alpha$ and $\mathrm{Mg} \mathrm{I} \lambda 2852$. The asymmetric tails are perhaps relics of the asymmetries observed at much earlier phases (Hanuschik \& Dachs 1987; Larson et al. 1987; Phillips \& Heathecote 1989), thus implying that ${ }^{57}$ Co and ${ }^{44} \mathrm{Ti}$, just like ${ }^{56} \mathrm{Co}$, are distributed asymmetrically.

Future observations using the HST should prove to be important in studying the evolution of the SN 1987A ejecta. Luo, McCray, \& Slavin (1994) predict that the expanding ejecta of SN 1987A will hit the circumstellar ring in $1999 \pm 3$; a recent model by Chevalier \& Dwarkadas (1995) shows that the interaction may start in $2005 \pm 3$. When the interaction begins, the supernova will become a bright ultraviolet source again. The HST data provide

unique wavelength coverage and spatial resolution and produce a large number of line fluxes which can be combined with models to extract a clearer picture of both the ejecta and the circumstellar ring.

This research is supported in part by NASA Grant GO 5652, NAGW 2905, and NSF Grant 9218035. We are grateful for conversations with R. Chevalier, N. Chugai, A. M. Khokhlov, B. Leibundgut, J. Spyromilio, E. J. Wampler, B. Wills, and S. E. Woosley.

\section{REFERENCES}

Arnaud, M. \& Rothenflug, R. 1985, A\&A Supl, 60, 425

Begeron, J. \& Kunth, D. 1980, A\&A, 85, L11

Bouchet, P., Danziger, I.J., Gouiffes, C., Della Valle, M., \& Moneti, E. 1993, in IAU Coll. 145 Supernovae and Supernova Remnants, ed. R. McCray and Z.-R. Wang, Cambridge Univ. Press, in press 
Chevalier, R. A. \& Dwarkadas, V. V. 1995, ApJ, 452, L45

Chugai, N. N. 1988, Astron. Zircular, No. 1525

Chugai, N. N. 1991a, Sov. Astron., 35, 171

Chugai, N. N. 1991b, Sov. Astron. Lett., 17, 400

Chugai, N. N. 1994, ApJ, 428, L17

Chugai, N. N., Danziger, I. J., Wampler, E. J., \& Wang, L. 1993, unpublished

Clayton, D. C., Leising, M. D., \& The, L.-S. 1992, Ap. J., 399, L141

Fransson, C., Casatella, A., Gilmozzi, R., Kirshner, R. P., \& Panagia, N. 1989, ApJ, 336, 429

Fransson, C., \& Kozma, C. 1993, ApJ, 408, L25

Fransson, C., Houck, J., \& Kozma, C. 1994, in IAU Coll. 145 Supernovae and Supernova Remnants, ed. R. McCray and Z.-R. Wang, Cambridge Univ. Press, in press

Hanuschik, R., \& Dachs, J. 1987, A\&A, 182, L29

Hashimoto, M., Nomoto, K., \& Shigeyama, T. 1989, A\&A, 210, L5

Jakobson, P., Macchetto, F., \& Panagia, N. 1993, ApJ, 403, 736

Jakobson, P., Jedrzejewski, R., Macchetto, F., \& Panagia, N. 1994, ApJ, L47

Kirshner, R. P., Sonneborn, G., Grenshaw, D. M., \& Nassiopoulos, G. E. 1987, ApJ, 320, 602

Kozma, C., \& Fransson, C. 1992, ApJ, 390, 602

Larson, H. P., Drapatz, S., Mumma, M. J. \& Weaver, H. A. 1987, in ESO Workshop on the SN 1987A, Ed. I. J. Danziger, ESO Garching, p147

Laher, R. R., \& Gilmore, F. R. 1989, J. Phys. Chem. Ref. Data, 19, 277

Li, H., \& McCray, R. 1992, ApJ, 387, 309

Li, H., \& McCray, R. 1995, ApJ, 441, 821

Lucy, L. B., Danziger, I. J., Gouiffes, C., \& Bouchet, P. 1989. In IAU Coll. No. 120 Structure and Dynamics of the Interstellar Medium, ed. G. Tenorio-Tagle, M. Moles, J. Melnick, P 164. New York: Springer-Verlag

Lucy, L. B., Danziger, I. J., Gouiffes, C., \& Bouchet, P. 1991, in Supernovae, ed. S. E. Woosley (New York:Springer), p82

McCray, R. 1993, Annu. Rev. Astron. Astrophys., 31, 175

Luo, D., McCray, R., \& Slavin, J. 1994, ApJ, 430, 246 
Oke, J. B., \& Lauer, T. R. 1979, ApJ, 230, 360

Osterbrock, D. E. 1989, Astrophysics of Gaseous Nebulae and Active Galactic Nuclei, University Science Books

Panagia, N., Gilmozzi, R., Clavel, J., Barylak, M., Gonzales, Riesta, R.1987, A\&A, 177, L25

Panagia, N., Scuderi, S., Gilmozzi, R., Challis, P. M., Garnavich, P. M., \& Kirshner, R. P. 1995, ApJ, in press

Péquignot, D., Petitjean, P., \& Boisson, C. 1991, A\&A, 251, 680

Phillips, M. M., \& Heathecote, S. R. 1989, PASP, 101, 137

Phillips, M. M., \& Williams, R. E. 1991, in Supernovae, ed. S. E. Woosley (New York:Springer). p36

Plait, P.C., Lundqvist, P., Chevalier, R. A., \& Kirshner, R. P. 1995, ApJ, 439, 730

Pun, et al. 1996, in preparation

Pun, C. S. J., Kirshner, R. P., Sonneborn, G., Challis, P., Nassiopoulos, G., Arquilla, R., Crenshaw, D. M., Shrader, C., Teays, T., Cassatella, A., Gilmozzi, R., Talavera, A., Wamsteker, W., Fransson, C., \& Panagia, N. 1995, ApJS, 99, 223

Spyromilio, J., \& Pinto, P. A. 1991, In Proc. ESO/EIPC Supernovae Workshop, ed. I. J. Danziger (Garching, ESO)

Spyromilio, J., Meikle, W. P. S., \& Allen, D. A. 1990, MNRAS, 242, 669

Swartz, D. 1991, in Supernovae, ed. S. E. Woosley (New York:Springer). p434

Thielemann, F. K., Nomoto, K., \& Hashimoto, M. 1995, ApJ, submitted

Timmes, F. X., Woosley, S. E., Hartmann, D. H, \& Hoffman, R. D. 1995, ApJ, in the press

Walborn, N. R., Phillips, M. M., Walker, A. R., and Elias, J. H. 1993, PASP, 105, 1240

Wampler, E. J., \& Richichi, A. 1989, A\&A, 217, 31

Wang, L., D’Odorico, S., Gouiffes, C., \& Wampler, E. J. 1992, IAUC 5449

Woosley, S. E. 1988, ApJ, 330, 218

Woosley, S. E., \& Hoffman, R. D. 1991, ApJ, 368, L31

Williams, R. E. 1982, ApJ, 261, 170

Xu, Y., \& McCray, R. 1991, ApJ, 375, 190 
Table 1. Summary of FOS Observations of SN 1987A

\begin{tabular}{|c|c|c|c|c|c|}
\hline Dataset & UT Date & Grating & Detector & Exposure & Position \\
\hline Y0WY0202T & $03 / 30 / 92$ & FOS/RD & G400H & 1500.0 & centered \\
\hline Y0WY0203T & $03 / 30 / 92$ & FOS/RD & G570H & 1500.0 & centered \\
\hline Y0WY0204T & $03 / 31 / 92$ & FOS/RD & $\mathrm{G} 780 \mathrm{H}$ & 1500.0 & centered \\
\hline Y0WY0102T & $04 / 01 / 92$ & FOS/BL & G130H & 1500.0 & centered \\
\hline Y0WY0103T & $04 / 02 / 92$ & FOS/BL & $\mathrm{G} 130 \mathrm{H}$ & 1500.0 & centered \\
\hline Y0WY0104T & $04 / 02 / 92$ & FOS/RD & G190H & 1500.0 & centered \\
\hline Y0WY0105T & $04 / 02 / 92$ & FOS/RD & G190H & 1500.0 & centered \\
\hline Y0WY0106T & $04 / 02 / 92$ & FOS/RD & $\mathrm{G} 270 \mathrm{H}$ & 1500.0 & centered \\
\hline Y0WY0107T & $04 / 02 / 92$ & FOS/RD & $\mathrm{G} 270 \mathrm{H}$ & 1500.0 & centered \\
\hline Y0WY0602T & $03 / 14 / 93$ & FOS/RD & $\mathrm{G} 400 \mathrm{H}$ & 1500.0 & centered \\
\hline Y0WY0603T & $03 / 14 / 93$ & FOS/RD & G570H & 1500.0 & centered \\
\hline Y0WY0604T & $03 / 14 / 93$ & FOS/RD & $\mathrm{G} 780 \mathrm{H}$ & 1500.0 & centered \\
\hline Y0WY5602T & $04 / 01 / 93$ & FOS/RD & G400H & 1500.0 & offcenter \\
\hline Y0WY5603T & $04 / 01 / 93$ & FOS/RD & G570H & 1500.0 & offcenter \\
\hline Y0WY5604T & $04 / 01 / 93$ & FOS/RD & G780H & 1500.0 & offcenter \\
\hline Y0WY0302T & $04 / 02 / 93$ & FOS/BL & G130H & 1500.0 & offcenter \\
\hline Y0WY0303T & $04 / 02 / 93$ & FOS/BL & G130H & 1500.0 & offcenter \\
\hline Y0WY0304T & $04 / 02 / 93$ & FOS/RD & G190H & 1500.0 & offcenter \\
\hline Y0WY0305T & $04 / 02 / 93$ & FOS/RD & $\mathrm{G} 190 \mathrm{H}$ & 1500.0 & offcenter \\
\hline Y0WY0306T & $04 / 02 / 93$ & FOS/RD & G270H & 1500.0 & offcenter \\
\hline Y0WY0307T & $04 / 02 / 93$ & FOS/RD & G270H & 1500.0 & offcenter \\
\hline Y0WY0402T & $04 / 03 / 93$ & FOS/RD & $\mathrm{G} 400 \mathrm{H}$ & 1500.0 & offcenter \\
\hline Y0WY0403T & $04 / 03 / 93$ & FOS/RD & G570H & 1500.0 & offcenter \\
\hline Y0WY0404T & $04 / 03 / 93$ & FOS/RD & G780H & 1500.0 & offcenter \\
\hline Y0WY0502T & $04 / 06 / 93$ & FOS/BL & G130H & 1500.0 & offcenter \\
\hline Y0WY0503T & $04 / 06 / 93$ & FOS/BL & G130H & 1500.0 & offcenter \\
\hline Y0WY0504T & $04 / 06 / 93$ & FOS/RD & G190H & 1500.0 & offcenter \\
\hline Y0WY0505T & $04 / 06 / 93$ & FOS/RD & G190H & 1500.0 & offcenter \\
\hline Y0WY0506T & $04 / 06 / 93$ & FOS/RD & G270H & 1500.0 & offcenter \\
\hline Y0WY0507T & $04 / 06 / 93$ & FOS/RD & G270H & 1500.0 & offcenter \\
\hline
\end{tabular}


Table 2. Observational Parameters

\begin{tabular}{lccccc}
\hline \hline FOS Grating & Detector & Wavelength Region $(\AA)$ & Dispersion $^{\mathrm{a}}$ & Resolution $^{\mathrm{b}}$ & Aperture \\
\hline G130H & Blue & $1140-1605$ & 0.25 & 1.1 & $0^{\prime \prime} .25 \times 2^{\prime \prime} .0$ \\
G190H & Red & $1600-2310$ & 0.36 & 1.5 & $0^{\prime \prime} .25 \times 2^{\prime \prime} .0$ \\
G270H & Red & $2230-3270$ & 0.51 & 2.1 & $0^{\prime \prime} .25 \times 2^{\prime \prime} .0$ \\
G400H & Red & $3250-4791$ & 0.74 & 3.1 & $0^{\prime \prime} .25 \times 2^{\prime \prime} .0$ \\
G570H & Red & $4200-6800$ & 1.08 & 4.1 & $0^{\prime \prime} .25 \times 2^{\prime \prime} .0$ \\
G780H & Red & $6500-8600$ & 1.43 & 5.1 & $0^{\prime \prime} .25 \times 2^{\prime \prime} .0$ \\
\hline
\end{tabular}

an units of $\AA /$ pix

${ }^{b}$ In units of $\operatorname{FWHM}(\AA)$. 
Table 3. Line List

\begin{tabular}{|c|c|c|c|c|c|c|c|}
\hline \multirow[b]{2}{*}{ Wavelength $(\AA)$} & \multirow[b]{2}{*}{ Flux $^{\mathrm{a}}$} & \multicolumn{2}{|c|}{1992 Mar 30} & \multicolumn{3}{|c|}{1993 Mar 14} & \multirow[b]{2}{*}{ Identification } \\
\hline & & Flux $^{b}$ & $\mathrm{FWHM}(\AA)$ & Flux $^{\mathrm{a}}$ & Flux $^{\mathrm{a}}$ & $\operatorname{FWHM}(\AA)$ & \\
\hline 2410.3 & 2.6 & 6.8 & 58.7 & 2.0 & 5.0 & 60.2 & Fe II (UV2) \\
\hline 2630.4 & 3.0 & 6.5 & 59.6 & 2.7 & 5.9 & 54.6 & Fe II (UV1) \\
\hline 2828.8 & 10.5 & 20.6 & 135.0 & 6.4 & 12.7 & 127.3 & Mg II $\lambda \lambda 2795,2802, \mathrm{MgIUV}$ \\
\hline 3314.5 & 1.8 & 3.1 & 50.0 & 2.4 & 4.1 & 45.0 & $\mathrm{Fe}$ II (1) \\
\hline 3476.4 & 2.1 & 3.5 & 39.0 & 2.7 & 4.4 & 45.1 & Fe II $(4,6)$, Fe I $(6)$ \\
\hline 3620.5 & 18.4 & 29.7 & 79.5 & 15.2 & 24.6 & 72.1 & $\mathrm{Bac}$ \\
\hline 3737.8 & 21.0 & 32.8 & 63.6 & 24.6 & 38.6 & 49.7 & {$[\mathrm{O}$ II $] \lambda \lambda 3726,3728$} \\
\hline $4229.3^{\mathrm{a}}$ & & & & & & & {$[\mathrm{Fe} \mathrm{II}](21 \mathrm{~F})$} \\
\hline $4335.7^{\mathrm{a}}$ & 41.4 & 55.9 & 282.1 & 50.4 & 68.0 & 284.0 & $\mathrm{H} \gamma,[\mathrm{Fe} \mathrm{II}](21 \mathrm{~F})$ \\
\hline $4456.3^{\mathrm{a}}$ & & & & & & & {$[\mathrm{Fe} \mathrm{II}](6 \mathrm{~F})$} \\
\hline 4554.3 & 13.5 & 17.4 & 41.3 & 13.9 & 18.0 & 39.3 & $\mathrm{Mg} \mathrm{I}] \lambda 4571$ \\
\hline 4701.9 & 1.5 & 1.9 & 52.9 & 3.0 & 3.8 & 60.0 & {$[\mathrm{Fe} \mathrm{II}](5 \mathrm{~F})$} \\
\hline 4863.8 & 20.7 & 25.4 & 86.2 & 22.2 & 27.3 & 75.1 & $\mathrm{H} \beta$ \\
\hline 5010.9 & 5.1 & 6.0 & 45.3 & 3.6 & 4.3 & 39.1 & {$[\mathrm{Fe} \mathrm{II}](4 \mathrm{~F})$} \\
\hline 5160.0 & 11.1 & 13.0 & 78.6 & 7.8 & 9.2 & 73.8 & {$[\mathrm{Fe} \mathrm{II}](18 \mathrm{~F}, 35 \mathrm{~F})$} \\
\hline 5277.0 & 9.9 & 11.5 & 93.7 & 6.4 & 7.3 & 83.6 & {$[\mathrm{Fe} \mathrm{II}](17 \mathrm{~F}, 18 \mathrm{~F}, 35 \mathrm{~F})$} \\
\hline 5685.2 & 9.0 & 9.9 & 118.2 & 9.3 & 10.3 & 107.6 & [Fe II] $(33 \mathrm{~F})$ \\
\hline 5895.2 & 20.1 & 21.5 & 118.7 & 20.1 & 21.6 & 119.2 & He I $\lambda 5876$, Na I $\lambda 5896$ \\
\hline 6294.3 & 17.4 & 17.8 & 99.9 & 19.1 & 19.6 & 115.7 & {$\left[\begin{array}{ll}\mathrm{O} & \mathrm{I}\end{array}\right] \lambda \lambda 6300,6364$} \\
\hline 6558.8 & 100.0 & 100.0 & 97.1 & 100.0 & 100.0 & 96.0 & $\mathrm{H} \alpha$ \\
\hline 7306.6 & 44.7 & 42.0 & 125.1 & 42.2 & 39.6 & 133.9 & {$[\mathrm{O}$ II $] \lambda 7320,[\mathrm{Ca} \mathrm{II}] \lambda 7300$} \\
\hline
\end{tabular}

${ }^{\mathrm{a}}$ Observed flux.

${ }^{\mathrm{b}}$ Dereddened flux.

${ }^{\mathrm{c}}$ These three lines are blended. 
Table 4. Maximum Observable Velocities for Strong Lines

\begin{tabular}{lrr}
\hline \hline \multicolumn{1}{c}{ Line } & 1992 Mar $^{\mathrm{a}}$ & $1993 \mathrm{Mar}^{\mathrm{a}}$ \\
\hline $\mathrm{H} \alpha$ & 11000 & 11500 \\
$\mathrm{H} \beta$ & 7200 & 6900 \\
{$[\mathrm{O} \mathrm{I}] \lambda 6300$} & 6000 & 5800 \\
$\mathrm{Mg} \mathrm{I}] \lambda 4571$ & 4000 & 3900 \\
{$[\mathrm{O} \mathrm{II}] \lambda \lambda 3726,3728$} & 4400 & 4750 \\
$\mathrm{Mg}$ I $\lambda 2852$ & 10000 & 10000 \\
Balmer Cont. & 3100 & 2700 \\
\hline
\end{tabular}

${ }^{\mathrm{a}}$ In units of $\mathrm{kms}^{-1}$. 


\section{Figure Captions}

Fig. 1... The slit orientation and position for each set of observations.

Fig. 2... a) The 1992 March 31 spectrum and the suggested line identifications; b) the 1993 March 14 spectrum. Both spectra are corrected for interstellar extinction assuming $E(B-V)=0.2 \mathrm{mag}$. The circumstellar lines are removed from the spectra.

Fig. 3... $\mathrm{H} \alpha$ and $\mathrm{H} \beta$ line profiles showing redshifted tails with velocities reaching 10000 $\mathrm{km} \mathrm{s}^{-1}$. The $\mathrm{H} \beta$ line is apparently asymmetric but the redshifted tail is corrupted by a neighboring iron line. Note that the narrow $\mathrm{H} \alpha, \mathrm{H} \beta$, and $[\mathrm{N} \mathrm{II}]$ lines are removed.

Fig. 4... The observed hydrogen Balmer continuum profiles (solid lines) and the corresponding model fits (dashed lines).

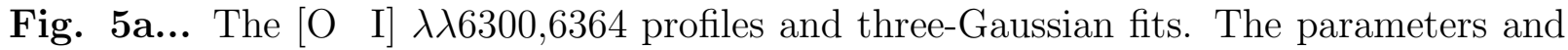
constraints employed are given in the text.

Fig. 5b... The $\left[\begin{array}{ll}\mathrm{O} & \mathrm{I}\end{array}\right] \lambda \lambda 6300,6364$ doublet decomposed by setting the ratio $R=3$. The decomposed line profiles are compared with the profiles of the Mg I] $\lambda 4571$ line for each observation. Note that the Mg I] $\lambda 4571$ lines are arbitrarily shifted upwards for clearity. The sum of the [O I] $\lambda 6300$ and [O I] $\lambda 6364$ profiles matches exactly the corresponding observed [O $\mathrm{I}$ I] $\lambda \lambda 6300,6364$ profile by definition (c.f. equation (3))

Fig. 6... The Mg I $\lambda 2852$ line is compared with the $\mathrm{H} \alpha$ and $\mathrm{Mg} \mathrm{I}] \lambda 4571$ lines. From top to bottom, the profiles are $\mathrm{H} \alpha, \mathrm{Mg}$ I $\lambda 4571$ and $\mathrm{Mg}$ I $\lambda 2852$. Both the $\mathrm{Mg} \mathrm{I} \lambda 2852$ and $\mathrm{H} \alpha$ lines show high velocity components reaching $10,000 \mathrm{~km} \mathrm{~s}^{-1}$, but the FWHM of the Mg I $\lambda 2852$ line is much larger than that of any other observed lines. 